This is an Accepted Manuscript (post print version) of an article published by Taylor \& Francis Group in Media Psychology on 18/12/2018, available online:

https://www.tandfonline.com/doi/full/10.1080/15213269.2018.1554494

\title{
Picture-perfect lives on social media: a cross-national study on the role of media ideals in adolescent well-being
}

Orpha de Lenne ${ }^{1,2}$, Laura Vandenbosch ${ }^{1}$, Steven Eggermont ${ }^{1}$, Kathrin Karsay ${ }^{3}$, and Jolien Trekels $^{1}$

Affiliations: ${ }^{1}$ School for Mass Communication Research, Faculty of Social Sciences, KU Leuven, Leuven Belgium; ${ }^{2}$ Research Foundation Flanders (FWO-Vlaanderen); ${ }^{3}$ Department of Communication, University of Vienna, Vienna, Austria

Email addresses: orpha.delenne@kuleuven.be; laura.vandenbosch@kuleuven.be;

steven.eggermont@kuleuven.be; kathrin.karsay@univie.ac.at; jolien.trekels@kuleuven.be

Please cite as follows: de Lenne, O., Vandenbosch, L., Eggermont, S., Karsay, K., \& Trekels, J. (2018). Picture-perfect lives on social media: a cross-national study on the role of media ideals in adolescent well-being. Media Psychology. doi: 10.1080/15213269.2018.1554494

Correspondence concerning this article should be addressed to Laura Vandenbosch, School for Mass Communication Research, KU Leuven, Parkstraat 45, B-3000 Leuven, Belgium, laura.vandenbosch@kuleuven.be 
A number of studies have suggested that social media use may be negatively related to adolescents' well-being. One explanation for this relation may lie in the internalization of various types of ideals displayed on social media; however, research supporting this reasoning is largely absent. The present survey study among 1,983 Austrian, Belgian, Spanish, and South Korean adolescents (aged 12-19, 49.7\% girls) addressed this gap and found that Instagram use was positively related to the internalization of professional, social, sexual, and romantic ideals, and Facebook use was positively related to the internalization of social and romantic ideals. In turn, the internalization of sexual ideals was related to poor mental well-being. Furthermore, Facebook use was also directly associated with poor mental well-being. Cross-national differences played an important role in that South Korea significantly differed from Austria, Belgium, and Spain in the relations between social media use, the internalization of social ideals, and poor mental well-being. We also found differences among Belgium, Austria, and Spain in the relations between social media use, the internalization of professional, social, and sexual ideals, and poor mental well-being.

Keywords: social media, Instagram, Facebook, internalization, media ideals, wellbeing, adolescents, cross-national study 


\section{Picture-perfect lives on social media: a cross-national study on the role of media ideals in adolescent well-being}

Social media represent an integral part of adolescents' daily media diet. For example, a Belgian report showed that almost 55\% use four social networking sites or more (Vanhaelewyn \& De Marez, 2017). Facebook is the most widely used, but photo-sharing platforms such as Instagram are increasingly popular (Lee, Lee, Moon, \& Sung, 2015). More than half of Belgian adolescents aged 15 to 19 years old spend at least 1 hour per day on Facebook and Instagram (Vanhaelewyn \& De Marez, 2017).

Scholars concerned about the implications of these practices have approached potential relations between social media use and mental well-being with opposing assumptions. Some research has assumed and found that intensity of social media use is related to better mental well-being (i.e., increased life satisfaction; Valenzuela, Park, \& Kee, 2009), while others have reported connection to reduced mental well-being (e.g., lower life satisfaction; Blachnio, Przepiorka, \& Pantic, 2016). Although different approaches towards the measuring of mental well-being and social media use partially clutter the comparison of studies, it is clear that we have a limited understanding of how adolescents develop a negative mental well-being following social media exposure. We conceptualize negative or poor mental well-being as experiencing symptoms of typical mental disorders such as depression and anxiety, during a limited time span (Rumpf, Meyer, Hapke, \& John, 2001).

In other domains producing mixed effects, the literature has argued that media effects may more often develop indirectly (through mediators) than directly (Vandenbosch \& Eggermont, 2012). It is possible, then, that the link between social media use and poor mental well-being may develop through the internalization of media ideals. Mass media have been shown to present superficial and narrow representations of societal ideals. For instance, sexual behaviors on television programs are often accompanied by positive outcomes whereas the 
negative health risks are rarely a part of this narrow narrative (e.g., Eyal, Kunkel, Biely, \& Finnerty, 2007). Moreover, professions are often well-paid and individuals are highly educated (Signorielli, 1997), thereby highlighting the importance of complying with these (educational and professional) ideals while disregarding the genuine happiness that individuals could derive from their educational and professional choices. These superficial interpretations of ideals are particularly prevalent on social media. "Posts" on social media are very positive and thus provide an idealized view of reality (Mendelson \& Papacharissi, 2010). These biased representations may change users' cognitions about which norms are important to attain (Thompson \& Stice, 2001). Drawing on social cognitive and sociocultural theory, the literature has suggested that norms regarding professional, social, sexual, and romantic ideals are prevalent on social media (Mendelson \& Papacharissi, 2010; Ramsey \& Horan, 2017) and may consequently be internalized, which could, in turn, negatively affect mental well-being. However, research theorizing and examining the internalization of these ideals as mediating variables is lacking. Therefore, the current study aims to explore the internalization of professional, social, sexual, and romantic media ideals as an explanatory factor in the relation between social media use and adolescents' poor mental well-being.

In addition, our study contributes to the literature in at least two other ways. First, it explored the potential importance of including different social media applications, i.e., Facebook vs. Instagram. Most studies on social media and well-being investigated social media in general (e.g., Vandenbosch \& Eggermont, 2012) or focused only on Facebook (e.g., Blachnio et al., 2016). However, as Facebook and Instagram are characterized by different features and user motivations (Sheldon \& Bryant, 2016), it may be important to take both Facebook and Instagram into account when studying the relations between social media use, the internalization of media ideals, and subsequent poor mental well-being. 
Second, this study used an intercultural sample of Austrian, Belgian, Spanish, and South Korean adolescents. Previous research indicates that culture affects social media content and motivations to use social media (e.g., Götzenbrucker \& Köhl, 2014). It thus seems meaningful to take cross-national differences into account when studying the relations between social media use, the internalization of media ideals, and poor mental well-being.

\section{Facebook and Instagram Use During Adolescence}

Social media intertwine with different adolescent developments. Adolescents' relational development, in which social acceptance is essential (Steinberg \& Morris, 2001), is connected to the fact that social media help to obtain information about others by investigating their profiles (Raacke \& Bonds-Raacke, 2008), a frequently performed practice according to research on both Facebook (Raacke \& Bonds-Raacke, 2008) and Instagram (Sheldon \& Bryant, 2016). The need for popularity is positively related to browsing others' profiles (Utz, Tanis, \& Vermeulen, 2012), suggesting that social media may inform adolescents about which behaviors are socially accepted and increase one's popularity. In addition, an important task during adolescence is to construct a stable identity (Steinberg \& Morris, 2001). Adolescents engage in a process of identity exploration in which they start to make important life decisions and reflect about themselves (Subrahmanyam \& Šmahel, 2011). Social media facilitate this process by allowing adolescents to engage in self-presentation practices and to choose which part of their identity they show online. Social media tools for expressing one's identity are sharing photos or adding textual comments in which relevant aspects of their self can be reflected on (Subrahmanyam \& Šmahel, 2011). Such self-presentation practices allow adolescents to experiment with their identity by sharing different parts of their personality and see how peers react on that (Subrahmanyam \& Šmahel, 2011). In this respect, the literature has specified that self-presentation is more important on Instagram than on Facebook (Sheldon \& Bryant, 2016). 
Given the prevalence of such seeking and sharing of information, it seems relevant to examine which types of messages prevail on social media platforms. Most information posted online represents success stories. Negative experiences or emotions are rarely shared (Mendelson \& Papacharissi, 2010). Teens explain that they dislike negative posts and avoid posting messages that represent drama (Madden et al., 2013). This focus on positive or ideal messages may be especially present within the utilitarian individualistic culture that typifies Western societies (Bellah, Madsen, \& Sullivan, 1985). Utilitarian individualism values success and idealizes self-made men or women who have attained accomplishments through hard work and commitment (Bellah et al., 1985). In contemporary society, these achievements mostly refer to academic performance (Luthar \& Becker, 2002), popularity (Utz et al., 2012), sexual attractiveness (Ramsey \& Horan, 2017), and romantic relationships (Furman, 2002).

On social media, this focus on ideals is reflected in adolescents' posting behavior. Teenagers post pictures of important academic events, such as graduation (e.g., Mendelson \& Papacharissi, 2010). They also share social activities. Pictures posted on Facebook (Mendelson \& Papacharissi, 2010) and Instagram (Hu, Manikonda, \& Kambhampati, 2014) commonly represent users with their friends at social events, such as parties. The number of online connections itself also conveys information about one's social status. The number of friends (Facebook) or followers (Instagram) is an indicator of popularity (Zywica \& Danowski, 2008).

Research has shown that messages regarding sexuality are prevalent and rewarded on social media; sexualized pictures receive more likes than other pictures (Ramsey \& Horan, 2017), and both teen girls and young women post pictures in flirtatious poses (e.g., showing cleavage) or sexy clothing (e.g., swimwear), particularly on Instagram (Ramsey \& Horan, 2017). Analogously, research reported an emphasis on romantic success, with $72.4 \%$ of male and $62.1 \%$ of female Facebook users showing their partner in profile pictures and $79.3 \%$ of male and $82.8 \%$ of female users displaying their relationship status (Papp, Danielewicz, \& 
Cayemberg, 2012). Once users are in a romantic relationship, this relationship tends to become the main focus in their social media posts (Mendelson \& Papacharissi, 2010).

\section{Social Media and the Internalization of Media Ideals}

Social cognitive theory posits that one can learn which social norms are socially accepted by observing the behavior of role models (e.g., peers, bloggers, celebrities) on social media, especially when it is rewarded (e.g., through likes) (Bandura, 2001). Sociocultural theory adds that this learning can result in the internalization of the learned norms or ideals (Thompson \& Stice, 2001). Although internalization processes have originally been theorized for body ideals, we introduce a broader perspective and suggest that internalization may also occur for other types of media ideals. We define this internalization as the extent to which an individual cognitively adopts a socially defined ideal as personal (Thompson \& Stice, 2001). This means that one will no longer perceive an ideal as imposed by others but will instead perceive it as a personal goal s/he should strive to obtain. Internalization thus differs from the mere adoption of social norms, as the norms become part of one's identity.

On social media, the superficial nature of professional, social, sexual, and romantic ideals is likely to prevail. Specifically, social media is typically characterized by positive stories in which the importance of popularity or professional and academic success is emphasized at the expense of genuine happiness with one's social or professional life (referred to as media ideals). Exposure to superficial representations of professional, social, sexual, and romantic ideals on social media may result in internalization. Such relations may especially occur among adolescents, as academic, social, sexual, and romantic pressures characterize their stage of development. During this period, parents and teachers express educational expectations (Luthar \& Becker, 2002), social relationships with peers gain importance (Steinberg \& Morris, 2001), sexual changes occur (Russell, 2005), and initial romantic experiences emerge (Furman, 2002). These changes could make adolescents more attentive and susceptible to related ideals 
displayed on social media. Some studies have indeed hinted at such internalization processes. Social media use has been related to cognitions and behaviors that refer to the importance of academic performance (Al-rahmi, Zeki, Alias, \& Saged, 2017), popularity (Zywica \& Danowski, 2008), sexual attractiveness (Vandenbosch \& Eggermont, 2012), and romantic relationships (Papp et al., 2012). Thus, we aimed to test the following hypothesis:

H1: Facebook and Instagram use is positively related to the internalization of professional, social, sexual, and romantic ideals among adolescents.

As Facebook is more textually oriented and Instagram focuses on visual messages (Lee et al., 2015), they likely generate different usage styles. Research argued that because of its image-driven nature, Instagram is more often used to express one's personal identity and engage in self-promotion strategies than Facebook (Lup, Trub, \& Rosenthal, 2015; Sheldon \& Bryant, 2016). Facebook allows users to share a variety of messages, such as YouTube videos or news articles, whereas Instagram is mainly an opportunity to share content produced by users themselves. On Instagram, it is also common to follow people one does not know personally, such as celebrities (Lee et al., 2015; Lup et al., 2015). Findings suggest that users who are regularly exposed to the ideal lives of famous people tend to believe that others are more successful (Lup et al., 2015). Given these different user styles, it is possible that Instagram users are more often exposed to ideal messages than Facebook users. Thus, we hypothesized:

H2: The relations between Instagram use and the internalization of professional, social, sexual, and romantic ideals among adolescents are stronger than the relations between Facebook use and the internalization of those ideals.

\section{Internalization and Adolescents' Poor Mental Well-Being}

Internalization can be linked to decreased mental well-being through a process of social comparison. Adolescents who have internalized media ideals are more likely to compare their own performances to the accomplishments of others (Tiggemann \& Slater, 2014). Social media 
facilitate upward comparison, i.e., comparing oneself to someone who performs better, which can decrease well-being when dissimilarity is experienced between one's own accomplishments and the successes of others (Tiggemann \& Slater, 2014).

Mental well-being is particularly vulnerable during adolescence, when adolescents undergo developmental and psychological changes which often cause symptoms of depression (Steinberg \& Morris, 2001). As such, they may be more susceptible to the negative outcomes of the internalization of media ideals. Empirical research seems to support this. Research on appearance ideals among teenage girls has indicated that thin-ideal internalization leads to more symptoms of depression (Stice \& Bearman, 2001). Although no research has investigated the links between the internalization of professional, social, sexual, and romantic ideals and poor mental well-being, research on closely related processes suggest such associations may occur. For instance, pressure to attain academic ideals has been shown to result in symptoms of depression and anxiety among adolescents (Luthar \& Becker, 2002). Similarly, sexual performance pressure is related to negative well-being outcomes such as sexual dysfunctional problems (i.e., sexual problems such as premature ejaculation, erectile disorders, lack of sexual desire etc.; McCabe, 2005). Overall, the literature suggests that the internalization of media ideals can lead to decreased well-being, and thus we aimed to test the following hypothesis:

H3: The internalization of professional, social, sexual, and romantic ideals is positively related to poor mental well-being among adolescents.

Together, $\mathrm{H} 1$ and $\mathrm{H} 3$ assume a mediation model for the relations between Facebook and Instagram use and poor mental well-being. Although no research has investigated the internalization of professional, social, sexual, and romantic ideals as mediating processes, previous studies investigating beauty ideals indeed suggest that internalization can be an important mediator when investigating the relations between social media use and negative well-being outcomes (e.g., Vandenbosch \& Eggermont, 2012). As such, we hypothesized: 
H4: The positive relations between Facebook and Instagram use and poor mental wellbeing are mediated by the internalization of professional, social, sexual, and romantic ideals.

\section{Cross-national Differences}

Cultural differences in social media behavior are often studied by differentiating between individualistic and collectivistic cultures (Hofstede, 2001). Individuals from a collectivistic culture focus more on social interactions when using social media, whereas individuals from an individualistic culture focus more on self-promotion. For example, research comparing Austrian and Thai users indicates that Austrians mainly used social media to express themselves and construct their identity, compared to Thai users, who focused more on intensifying close relationships (Götzenbrucker \& Köhl, 2014). Similarly, several studies found that Americans focus on disclosing personal information and engaging in positive selfpresentation when using social media, whereas South Koreans attach more importance to social support and developing committed relationships (e.g., Kim, Sohn, \& Choi, 2011).

Although these findings provide important insights about cultural differences regarding social media use, they disregard the fact that not every country has a distinct individualistic or collectivistic orientation. Social media user differences could also occur between countries with less pronounced levels of individualism vs. countries with higher scores. Indeed, research highlights subtle differences and found that countries with lower levels of individualism focus more on connecting with others than countries with a distinct individualistic orientation (Alarcón-del-Amo, Gómez-Borja, \& Lorenzo-Romero, 2015). For example, Spanish users engage more actively with others on social media than their Dutch counterparts by commenting on posts, tagging friends in photos, and sending public messages to interact with their connections. Countries with less pronounced individualism scores may be less inclined to act as individuals and be more social, thus focusing on relations while using social media, 
compared to distinct individualistic cultures. The current study aims to challenge this individualistic-collectivistic dichotomy by studying countries with different levels of individualism or collectivism (i.e., Belgium as a highly individualistic country, Austria and Spain as individualistic countries with a less pronounced individualistic orientation, and South Korea as a collectivistic country; Hofstede, 2001) and thus takes into account a more nuanced perspective regarding social media variations across countries.

Besides their differences in levels of individualism and related social media habits, these countries may also be particularly interesting with regard to the studied ideals. Countries that score high on masculinity may be more susceptible to internalizing ideals as masculine societies heavily focus on achievements and success (Hofstede, 2001). This focus on being the best could be reflected in finding it important to attain professional, social, sexual, and romantic ideals. Austria, Belgium, Spain, and South Korea all have different masculinity scores, with Austria being a very masculine society, South Korea and Spain being feminine societies, and Belgium being a moderate masculine society (Hofstede, 2001). These differences in societal masculinity could translate into differences in the extent to which individuals have internalized these ideals and how they subsequently affect mental well-being.

Given the differences regarding levels of individualism and masculinity in Austria, Belgium, Spain, and South Korea, these countries may be particularly relevant for examining links between social media use, the internalization of different media ideals, and poor mental well-being. Because the current literature does not indicate a clear direction as to how countries with different levels of masculinity, individualism, or collectivism may differ with regard to these links, we aimed to examine the following research question:

RQ1: Are there differences in the relations between Facebook use, Instagram use, the internalization of professional, social, sexual, and romantic ideals, and adolescents' poor mental well-being among Austria, Belgium, Spain, and South Korea? 


\section{Method}

\section{Sample and Procedure}

Data were collected between February and May 2017 in Austria, Belgium, Spain, and South Korea as part of a large-scale cross-sectional study (the Intercultural Study Project, ISP ${ }^{1}$ ). Ethical approval was received from the ethical committees of each host university. A translation-backward translation method was used to translate the questionnaire in all countries except for Spain. In Spain, two researchers translated the questionnaire through a two-step procedure. In a first step, each of the researchers translated half of the questionnaire. In a second step, they reviewed the translated questionnaire in order to further standardize terms. Special attention was also given in each country to align the wording of the scales to adolescents' daily living environment. Convenience sampling was used to recruit schools. Selection was based on a list of schools that was constructed by the researchers or was provided by the government in some of the countries. Schools were contacted through e-mail and/or by telephone until a substantial number of schools agreed to participate in order to guarantee a minimum of 300 participants. Seven schools in Austria, eleven in Belgium, four in South Korea, and five in Spain participated. Before participating, parental active consent was obtained. Paper-andpencil surveys were filled out during class hours, and researchers were present during data collection. Anonymity and confidentiality was assured during participation.

A total of 1,983 adolescents ( $49.7 \%$ girls) aged 12 to 19 years old $(M=14.41$ years, $S D$ $=1.08)$ completed the questionnaire. The sample was distributed across the four countries as follows: Austria: $n=368,18.6 \%$ (54.1\% girls; 13 to 19 years old); Belgium: $n=675,34.0 \%$ (43.6\% girls; 12 to 16 years old); Spain: $n=564,28.4 \%$ (54.6\% girls; 13 to 17 years old); South Korea: $n=376,19.0 \%$ (49.1\% girls; 12 to 15 years old). Table 1 summarizes age

\footnotetext{
${ }^{1}$ The current paper uses data that are part of a larger intercultural study project that examines links between media usage and well-being among adolescents in four different countries. More information about the study project can be obtained by sending an email to the first author.
} 
differences and revealed that Belgian adolescents were significantly younger than Austrian, Spanish, and South Korean adolescents and South Korean adolescents were significantly younger than Austrian and Spanish adolescents $\left(F(24)=93.55, p<.001, \eta p^{2}=.29\right)$.

\section{Measures}

Facebook and Instagram use. Participants reported how much time they spent per day on Facebook or Instagram on a scale ranging from 1 (= never use it to less than 10 minutes) to 7 (= more than 6 hours).

Internalization of professional, social, sexual, and romantic ideals. Internalization scales were inspired by prior research among adolescents that investigated academic (DeCarlo \& Luthar, 2000), social (LaFontana \& Cillessen, 2002), sexual (Vandenbosch, van Oosten, \& Peter, 2017), and romantic (Sprecher \& Metts, 1989) ideals. After creating a draft version, four media expert researchers evaluated the appropriateness and wording of the items. Next, a panel of 17 college students from the KU Leuven were consulted to evaluate the wording of the items. The items were adapted according to the recommendations of the media expert researchers and the panel of college students.

Each scale contained two items using Likert scales ranging from 1 (= totally not important) to 10 (= totally important). Respondents were asked how important each item was to them personally. The internalization of professional ideals $(r=.40, p<.001)$ was measured by the items "Obtaining good results at school" and "Obtaining a future profession in which you will make a lot of money." The internalization of social ideals $(r=.47, p<.001)$ was measured by "Being a person with whom many peers want to hang out with" and "Excelling in extracurricular activities like hobbies but also shining at parties and social events, like dance performances or (sports) games." The internalization of sexual ideals $(r=.85, p<.001)$ was measured by "Having an exciting relationship with a boy-/girlfriend that includes a variety of physical affection (or touch)" and "Being/becoming good at physically affectionate acts, like 
kissing and cuddling." The internalization of romantic ideals $(r=.76, p<.001)$ was measured by "Being together with your soulmate, your true love" and "Having a relationship in which the love is so true that it conquers all troubles." As the internalization scales are newly developed measures, a confirmatory factor analysis (CFA) was conducted using Mplus to validate the proposed factor structure. The proposed factor structure showed a good fit with the data: $\chi^{2}(14)=75.58, p<.001$, RMSEA $=.05(90 \%$ CI: $.04 / .06)$, CFI $=.99$, TLI $=.98$, SRMR $=.02$. For descriptive purposes, new variables were created by averaging the item scores. Higher scores indicate a higher level of internalization for each variable.

Poor mental well-being. The Mental Health Inventory (MHI-5) was used (Rumpf et al., 2001). This scale asks participants how they felt during the past two weeks and contains five items, such as "I have been a very nervous person" and "I felt downhearted and blue." Items were rated on a scale ranging from 1 (= none of the time) to 6 (= all the time). Item two ("I felt calm and peaceful") and item five ("I have been a happy person") were rescaled. Principal components analysis (PCA) ensured the one-factor dimension of the scale (eigenvalue $=2.59$, explained variance $=51.74 \%, \alpha=.76)$. A new variable was created by averaging the item scores. Higher scores indicate poorer mental well-being.

Control variables. Gender $(1=$ male, $2=$ female $)$ and age were assessed as control variables, as previous research highlighted gender and age differences regarding social media use (e.g., Frison \& Eggermont, 2016a).

\section{Analytical Strategy}

Data were checked for normality. Skewness values were found to be below 3 and kurtosis values below 10 (Kline, 2011), confirming that data were normally distributed. Descriptive statistics and correlation analyses were calculated. Cultural differences in descriptive analyses were explored by conducting a MANOVA analysis. Before testing the hypothesized main model (see Figure 1), measurement invariance (i.e., invariance of factor 
loadings; Mplus) of the novel internalization scales was assessed by testing whether respondents in different countries responded to the internalization measures in the same manner. A configural model (multigroup model with no equality constraints) was compared to a metric model (equality constraints for factor loadings) using a $\chi^{2}$-difference test.

The main model (H1 and H3) was tested using structural equation modeling (Mplus). Instagram use, Facebook use, age, and gender were entered as manifest variables, while the internalization constructs and poor mental well-being were entered as latent variables. Control variables were modeled to predict the internalization constructs and mental well-being, while covariances with Facebook and Instagram use were included. Covariances between the latent internalization constructs were also modeled. Predictive pathways as shown in Figure 1 were modeled and indirect associations of Facebook and Instagram use on poor mental well-being via the internalization of the different ideals were calculated (H4). The following indices and criteria of acceptable fit were considered (West, Taylor, \& Wu, 2012): RMSEA $<.08$, CFI > .90, TLI $>.90$ and SRMR $<.08$. To test $\mathrm{H} 2$, difference parameters calculating the difference between pathways were added to the model.

To test RQ1, the structural invariance (invariance of pathways between media variables, internalization constructs, and poor mental well-being) of the hypothesized model was tested using a $\chi^{2}$-difference test to compare an unconstrained multigroup model (all paths of interest are set to be different across countries) to a constrained multigroup model (all paths of interest are specified to be the same across countries). Difference parameters were further added to determine which pathways differed between the four countries.

The significance of the examined pathways was assessed with bootstrapping (95\% biascorrected bootstrapped confidence intervals; 1000 bootstrapped samples). Confidence intervals and $\mathrm{p}$ values of standardized model results were reported. Full information maximum likelihood was used in Mplus to address missing data (Muthén \& Muthén, 2017). 


\section{Results}

\section{Descriptive statistics}

Table 2 displays the descriptive statistics and zero-order correlations. On average, adolescents reported spending more time on Instagram (31-60 minutes) than on Facebook (1030 minutes) per day. They also reported having internalized professional, social, and romantic ideals to a rather high extent. Respondents expressed moderate levels of internalization of sexual ideals. The average experienced poor mental well-being was rather low, indicating that adolescents felt relatively good during the two weeks before completing the survey.

Country differences were explored by performing a MANOVA analysis (see Table 1), including Facebook use, Instagram use, the internalization scales, poor mental well-being, and age, $F(24)=93.55, p<.001, \eta p^{2}=.29$. The most important results were that Belgian and South Korean adolescents used Facebook more frequently than their Austrian and Spanish counterparts. On the other hand, South Koreans used significantly less Instagram than Austrian, Belgian, and Spanish teens. Results further showed that South Koreans had internalized social ideals to a significant higher extent than Austrian, Belgian, and Spanish adolescents. The internalization of sexual ideals was the highest in Austria and Spain. Finally, no differences were found for the internalization of professional ideals and poor mental well-being.

\section{Measurement Invariance}

Measurement invariance was tested by performing multiple group analyses (see Table 3) (Byrne, 2013). First, the internalization scales showed a good model fit when tested in each country separately. Next, invariance tests were conducted: configural invariance (model 1), metric invariance (model 2) and partial metric invariance (model 3 and model 4). The $\chi^{2}$ difference test for models 1 and 2 was significant $(p<.001)$, indicating variance. We aimed to establish partial invariance in a next step by releasing the factor loadings with the highest modification indices (Byrne, 2013; Muthén \& Muthén, 2017). Equality constraints for the 
internalization of professional ideals in South Korea were released. After this adaptation, the $\chi^{2}$-difference test for models 1 and 3 was again significant $(p<.01)$. Therefore, equality constraints for the internalization of romantic ideals in South Korea and Austria were released. After this second adaptation, the $\chi^{2}$-difference test for models 1 and 4 was not significant, and thus partial measurement invariance was obtained. In short, the measurement of the internalization of professional ideals is variant in South Korea and the measurement of the internalization of romantic ideals is variant in South Korea and Austria.

\section{Testing the Hypothesized Model Across Countries}

Figure 1 displays the results for the hypothesized model that was tested using the full sample and showed a good fit: $\chi^{2}(87)=724.60, p<.001$, RMSEA $=.06$ (90\% CI: $\left..06 / .07\right)$, $\mathrm{CFI}=.94, \mathrm{TLI}=.90, \mathrm{SRMR}=.04$. Facebook use related positively to the internalization of social ideals, and romantic ideals. No significant relations were found between Facebook use and the internalization of professional or sexual ideals. As such, H1 was only partially confirmed regarding Facebook use.

Supporting H1 regarding Instagram use, the use of this social media platform related positively to the internalization of professional ideals, social ideals, sexual ideals, and romantic ideals. The difference parameters further indicated that the paths from Facebook to the internalization of professional $(p<.05)$ and sexual ideals $(p<.001)$ significantly differed from the paths from Instagram to the internalization of professional and sexual ideals. H2 was thus only confirmed for professional and sexual ideals but not for social and romantic ideals.

Partially confirming $\mathrm{H} 3$, the internalization of sexual ideals was positively related to poor mental well-being. No significant associations were found between the internalization of professional, social, and romantic ideals and poor mental well-being. A direct positive relation was found between Facebook use and poor mental well-being. No significant direct relation was found between Instagram use and poor mental well-being. 
No significant indirect associations were found between Facebook use, the internalization of the different ideals and poor mental well-being. The indirect relation between Instagram use, the internalization of sexual ideals, and poor mental well-being was found to be significantly positive, $\beta=.03, B=.01, S E=.00, p<.05,95 \%$ CI [.01 / .05]. No significant indirect relations were found between Instagram use and the internalization of professional, social, and romantic ideals and poor mental well-being. H4 was thus only confirmed for the internalization of sexual ideals in the relation between Instagram and poor mental well-being.

\section{Structural Invariance Analysis to Test for Country Differences}

To test differences in the proposed relations between countries (RQ1), we conducted structural invariance tests. First, the full model was tested in each country separately and had a good model fit in all countries (see Figures 2-5 for model results per country). Next, the $\chi^{2}-$ difference test, comparing the unconstrained multigroup model with the constrained multigroup model, was significant, indicating variance had occurred (see Table 3). Thus, a path-by-path analysis was conducted by adding difference parameters to identify the source of that variance. No differences were found for the relations between Instagram use and the internalization of professional, sexual, and romantic ideals, between Facebook use and the internalization of romantic ideals, and between all of the internalization variables and poor mental well-being. Pathways that showed to be variant across countries are discussed below (see also Table 4 for an overview of variant paths).

Instagram use and the internalization of social ideals. Instagram use is not related to the internalization of social ideals among South Korean adolescents but was significantly and positively related to the internalization of social ideals in the other countries.

Instagram use and poor mental well-being. The association between Instagram use and poor mental well-being in South Korea differed significantly from that association in 
Austria and Belgium. This relation was significantly negative in South Korea but not significant (and positive) in Austria and Belgium.

Facebook use and the internalization of professional ideals. This positive relation between Facebook use and the internalization of professional ideals differed significantly between Belgium and Austria and was significant in Austria but not in Belgium.

Facebook use and the internalization of social ideals. The positive relation between Facebook use and the internalization of social ideals differed significantly between South Korea and Belgium and Spain and between Austria and Spain. The relation was significant in Austria and South Korea but not in Belgium and Spain.

Facebook use and the internalization of sexual ideals. This positive relation between Facebook use and the internalization of sexual ideals differed significantly between Belgium and Spain and was significant in Belgium but not in Spain.

Facebook use and poor mental well-being. The positive relation between Facebook use and poor mental well-being differed significantly between Belgium and Spain. This direct relation was found to be significantly positive in Spain but not in Belgium.

\section{Discussion}

The current study provided novel insights into the relations between social media use and adolescents' poor mental well-being by proposing the internalization of professional, social, sexual, and romantic ideals as explanatory processes. The study offered a cross-cultural perspective on these relations and further explored the potential importance of including both Facebook and Instagram. The results showed different relations between adolescents' Facebook and Instagram use and the internalization of these media ideals. Only the internalization of sexual ideals was related to poor mental well-being. Additionally, Facebook use was directly linked to poor mental well-being. Differences between countries emphasized that several paths did not occur in all countries. These results contribute to understanding the 
explanatory role of the internalization of different media ideals in the relation between social media use and adolescents' poor mental well-being in four ways.

First, this study is one of the first to consider different media ideals, as most research focuses on appearance ideals (e.g., Tiggemann \& Slater, 2014) when studying (social) media effects. The findings on Facebook and Instagram use and the internalization of professional, social, sexual, and romantic ideals indicate that adolescents internalize different ideals when using social media. Facebook use was positively related to the internalization of social and romantic ideals but not to the internalization of professional and sexual ideals. In addition, Instagram use was positively related to the internalization of professional, social, sexual, and romantic ideals. Hypothesis 1 was thus partially supported. The reported relations with Facebook use are in line with previous research reporting that social and romantic relationships are the main subjects of Facebook pictures (Mendelson \& Papacharissi, 2010). The results for Instagram further coincide with its status as an excellent platform for adolescents to learn from observing idealized role models (Bandura, 2001; Lee et al., 2015; Sheldon \& Bryant, 2016). Thus, this study extends the literature by demonstrating that adolescents adopt a wide range of societal ideals from social media platforms. Future research should thus take this diversity of ideals into account when studying social media use.

Second, this study included different social media applications and indicated how their features may interact with the adoption of different ideals. We found differences in the relations between Facebook and Instagram use and the internalization of professional and sexual ideals. Instagram use was positively related to the internalization of professional and sexual ideals, whereas no significant relations were found for Facebook use and these ideals. Hypothesis 2 investigating the differences between Facebook and Instagram was thus supported for professional and sexual ideals. These findings can possibly be explained by Instagram's focus on self-presentation (Lee et al., 2015; Sheldon \& Bryant, 2016), which encourages users to 
display (biased) content and be exposed to messages on professional and sexual ideals more frequently than on Facebook. The finding regarding sexual ideals is also in line with existing research claiming that Instagram is a more sexualized media platform than Facebook (Ramsey \& Horan, 2017). However, at the same time, the results also indicate similarities between Facebook and Instagram regarding the internalization of social and romantic ideals. This is not surprising, as Facebook and Instagram, despite their differential focus on self-presentation, are still both social media platforms that encourage the development and maintenance of social relationships. Future research could further explain these findings by studying the culture and norms of different social media platforms and investigating why adolescents post certain messages on specific platforms. Content analyses exploring the prevalence of the different ideals may also be necessary to gain a better understanding of these results.

Furthermore, the variance in the internalization constructs may only be partly explained by social media use, as personal goals are also shaped by other socialization agents, such as parents and peers. Parents play an important role in the development of professional, social, sexual, and romantic goals. Educational expectations from parents are highly present during adolescence (Luthar \& Becker, 2002). Parent-child closeness also determines sexual and romantic relationships and connections with peers (Furman, 2002). Furthermore, adolescents spend substantial time with their peers (Steinberg \& Morris, 2001). Research indicates that teenagers talk to each other about career-related issues (Kracke, 2002) and influence each other's ideas about sexualized relationships (Cavanagh, 2007). These conversations can reinforce the importance of attaining the discussed ideals. Peers also determine adolescents' conceptions about idealized romantic relationships (Cavanagh, 2007). As such, peers could have an impact on the internalization of different societal ideals.

Third, this study is the first to study the internalization of professional, social, sexual, and romantic ideals as mediators in the relation between social media use and poor mental well- 
being. The internalization of sexual ideals was positively related to poor mental well-being; in addition, an indirect relation was found between Instagram use, the internalization of sexual ideals, and mental well-being. Previous research has already noted that sexual performance anxiety is related to sexual dysfunction (McCabe, 2005). The current study adds that Instagram use is related to a decreased mental well-being through the internalization of sexual ideals. This may not be surprising, given the importance of sexuality during adolescence (Russell, 2005) and the prevalence of sexualized photos on Instagram (Ramsey \& Horan, 2017). No significant results were found for the links between the internalization of professional, social, and romantic ideals and adolescents' poor mental well-being. Hypothesis 3 was thus only partially confirmed. No other indirect associations were found to be significant. Hypothesis 4 was thus also only partially confirmed as only the internalization of sexual ideals was a mediator in the relation between Instagram use and poor mental well-being.

Potentially, the internalization of some ideals may only affect mental well-being in a later phase of life. More precisely, adolescents are still in a phase in which it is expected that they are working toward obtaining professional and romantic goals. During emerging adulthood, the initiation of a long-term committed relationship as well as the choice of an academic and professional trajectory (Arnett, 2007) are central developmental tasks. As such, emerging adults who have internalized demanding professional and romantic goals during adolescence may especially encounter pressure to obtain their personal goals and, subsequently, develop a decreased mental well-being. Future longitudinal research may test these suggestions to examine the development of the associations between internalization and poor mental well-being from adolescence to emerging adulthood.

Related to this explanation is the measurement of the internalization variables. It is possible that these scales were not fully aligned to adolescents' daily living environment. The internalization of professional ideals included for example a career-oriented item (long-term 
goals) while adolescents may be more focused on short-term goals, i.e., academic achievements. Future research should further optimize these internalization scales so that they are attuned to ideals that are also relevant on a short-term basis.

In addition, the rather general measure of poor mental well-being could also explain these null results. It may be more relevant to investigate well-being outcomes that are specifically related to the different ideals, such as academic performance pressure (Luthar \& Becker, 2002), need for popularity (Zywica \& Danowski, 2008), sexual dysfunction (McCabe, 2005), and decreased relationship satisfaction (Papp et al., 2012).

It is also important to acknowledge that we only measured social media usage. Research has argued that merely measuring usage oversimplifies the possible effects of social media on adolescents' well-being (Frison and Eggermont, 2016b), which might explain several of the non-significant findings on poor mental well-being. Future research should examine specific social media behaviors and assess to which content adolescents are exposed on social media. Moreover, it should also be noted that some participants in our study did not use social media overall or only to a rather low extent compared to their peers. Future studies should consider more in-depth what this means for the internalization of ideals and poor mental well-being.

Finally, it is possible that exposure to media ideals on social media is not necessarily negatively related to mental well-being. If an ideal is perceived as attainable, individuals can feel inspired which in turn can generate positive outcomes towards well-being (Lockwood \& Kunda, 1997). This possibility thus indicates that the internalization of ideals may not affect mental well-being or even positively affect mental well-being because of inspiration effects.

Fourth, we found cross-national differences in the relations among Facebook and Instagram use, the internalization of the different ideals, and poor mental well-being (RQ1). In line with the collectivistic-individualistic differentiation (Hofstede, 2001), South Korea differed significantly in several relations from Austria, Belgium, and Spain. As collectivistic 
cultures highly value social interactions, it is no surprise that South Koreans reported high scores of internalizing social ideals (Kim et al., 2011). This focus on social ideals was reflected in their Facebook use but not in their Instagram behavior, as the relation between Instagram use and the internalization of social ideals was significant in the other countries but not in South Korea. South Koreans reported rarely using Instagram, which could indicate that they find Facebook a more favorable platform than Instagram to post or learn about social norms and ideals. This low Instagram use may also explain the rather surprising negative relation between Instagram use and poor mental well-being, which was significant in South Korea but not in Austria and Belgium. Possibly, because of their low overall Instagram usage, South Koreans may be less likely to post or be exposed to ideal messages, which might explain why lower Instagram use is related to poorer mental well-being. Future content analyses are recommended to gain a better insight into which messages South Koreans post on Instagram.

Furthermore, given the different levels of masculinity and since Belgians have a more distinct individualistic orientation compared to Austrians and Spaniards, several differences were also found between Belgium and Austria/Spain. Although Spaniards reported to have internalized sexual ideals to a rather high extent, this was not reflected in their Facebook use, since the relation between Facebook use and sexual ideals was significant in Belgium but not in Spain. However, it is likely that the variance in the sexual internalization construct is already captured by Instagram use, as Spaniards indicated being fervent Instagram users. This explanation also coincides with the existing literature indicating that Instagram is an excellent platform on which to post sexualized messages (Ramsey \& Horan, 2017). We also found differences between Belgium and Spain in the relation between Facebook use and poor mental well-being, which was significant in Spain but not in Belgium. This is a rather remarkable finding, given the low Facebook use among Spanish adolescents and the high Facebook use reported by Belgian adolescents. These results may be explained by the different levels of 
individualism in Belgium and Spain. Existing literature found that individuals from moderately individualistic countries (e.g., Spain) more actively use social media to publicly communicate and share information about themselves compared to users from highly individualistic countries (e.g., the Netherlands; Alarcón-del-Amo et al., 2015). As such, it is likely that Spaniards more actively post about their successes compared to Belgians, which could explain the subsequent negative mental well-being among Spanish adolescents. In addition, the relation between Facebook use and the internalization of professional ideals was found to be significant in Austria but not in Belgium. Since both countries had internalized professional ideals to an equally high extent, Belgians may have shifted to other social media platforms, such as LinkedIn, to advertise their professional successes. Austria is also characterized as a highly masculine society that is heavily focused on achievements. According to Hofstede (2001), this focus on success is often reflected in their professional life. It is thus possible that Austrians post more often about their professional achievements on Facebook than Belgians. Future research should further investigate this reasoning. Finally, in addition to these differences between Belgium and the other countries, we also found differences between Austria and Spain in the relation between Facebook use and the internalization of social ideals. Since Austria and Spain did not significantly differ in their Facebook use or the internalization of social ideals, this is a rather surprising finding that warrants future investigation.

Overall, these results might indicate that the collectivistic-individualistic and masculine-feminine distinctions are insufficient to explain cross-national differences regarding social media use, internalization, and poor mental well-being and that there are other factors that should be taken into account. Aside from these differences, we also found similarities in the relations between Facebook use, Instagram use, and the internalization of the different ideals, which hint at the idea that some ideals are promoted to the same extent on social media platforms across nations and that users are equally susceptible to adopting them. This was true 
for the links between Instagram use and the internalization of professional, sexual, and romantic ideals and the link between Facebook use and the internalization of romantic ideals.

Several limitations must be considered. The cross-sectional design does not allow us to draw conclusions regarding causality. Further longitudinal and experimental research is needed. Results should also be generalized with caution as the current study is based on a convenience sample. Future research could consider using probability sampling. Furthermore, the scales for the internalization of professional, social, sexual, and romantic ideals are newly developed measures. Future research should further improve these novel scales. Measurement invariance and structural invariance results also indicated that the proposed relations should be generalized with caution, as there exist cultural differences. In addition, it should be noted that translation-backward translation was not used in Spain, which could bias the reported cultural patterns in our study. Moreover, response rates were not tracked in detail. We also have to acknowledge that the studied countries were in part chosen due to practical reasons and except for South Korea, mainly included European countries. It would be interesting to investigate the studied relations in other Asian countries or countries like the U.S. that heavily focus on achievements and ideals. Finally, some of the participants were early adolescents who might not have hit puberty. Future research should take pubertal status into account as this could affect results, especially for sexual and romantic ideals.

In short, the current study is one of the first to demonstrate the role of the internalization of professional, social, sexual, and romantic ideals in the relation between social media use and poor mental well-being among adolescents. The results highlight cross-national differences and the importance of differential social media features and their relation to the internalization of different media ideals. 


\section{References}

Alarcón-del-Amo, M.-C., Gómez-Borja, M.-Á., \& Lorenzo-Romero, C. (2015). Are the users of social networking sites homogeneous? A cross-cultural study. Frontiers in Psychology, 6(1127). doi:10.3389/fpsyg.2015.01127

Al-rahmi, W. M., Zeki, A. M., Alias, N., \& Saged, A. A. (2017). Social media and its impact on academic performance among university students. The Anthropologist, 28, 52-68. doi:10.1080/09720073.2017.1317962

Arnett, J. J. (2007). Emerging adulthood: What is it, and what is it good for? Child Development Perspectives, 1, 68-73. doi:10.1111/j.1750-8606.2007.00016.x

Bandura, A. (2001). Social cognitive theory of mass communication. Media Psychology, 3, 265-299. doi:10.1207/S1532785XMEP0303_03

Bellah, R. N., Madsen, R., \& Sullivan, W. M. (1985). Habits of the heart: Individualism and commitment in American life. Berkeley, California: University of California press.

Blachnio, A., Przepiorka, A., \& Pantic, I. (2016). Association between Facebook addiction, self-esteem and life satisfaction: A cross-sectional study. Computers in Human Behavior, 55, 701-705. doi:10.1016/j.chb.2015.10.026

Byrne, B. M. (2013). Structural equation modeling with Mplus: Basic concepts, applications, and programming. New York, NY: Routledge.

Cavanagh, S. E. (2007). The social construction of romantic relationships in adolescence: Examining the role of peer networks, gender, and race. Sociological Inquiry, 77, 572600. doi:10.1111/j.1475-682X.2007.00207.x

DeCarlo, L. T., \& Luthar, S. S. (2000). Analysis and class validation of a measure of parental values perceived by early adolescents: An application of a latent class model for rankings. Educational and Psychological Measurement, 60, 578-591. doi:10.1177/00131640021970736 
Eyal, K., Kunkel, D., Biely, E.N., \& Finnerty, K.L. (2007). Sexual socialization messages on television programs most popular among teens. Journal of Broadcasting \& Electronic Media, 51, 316-336. doi: 10.1080/08838150701304969

Frison, E., \& Eggermont, S. (2016a). Gender and Facebook motives as predictors of specific types of Facebook use: A latent growth curve analysis in adolescence. Journal of Adolescence, 52, 182-190. doi:10.1016/j.adolescence.2016.08.008

Frison, E., \& Eggermont, S. (2016b). How Facebook makes teens (un)happy: Understanding the relationships between Facebook use and adolescents' well-being. (KU Leuven. Nieuwe reeks van doctoraten in de sociale wetenschappen en in de sociale en culturele antropologie 302). Leuven, Belgium: KU Leuven. Faculteit Sociale wetenschappen.

Furman, W. (2002). The emerging field of adolescent romantic relationships. Current Directions in Psychological Science, 11, 177-180. doi:10.1111/1467-8721.00195

Götzenbrucker, G., \& Köhl, M. (2014). Online relationship management, friendship cultures, and ego-networks of young people in Thailand and Austria. Asia Europe Journal, 12, 265-283. doi:10.1007/s10308-014-0384-8

Hofstede, G. (2001). Culture's consequences: Comparing values, behaviors, institutions, and organizations across cultures. Thousand Oaks, California: Sage.

Hu, Y., Manikonda, L., \& Kambhampati, S. (2014). What we Instagram: A first analysis of Instagram photo content and user types. Presented at the International AAAI Conference on Weblogs and Social Media, Ann Arbor, Michigan. Palo Alto, California: AAAI Press.

Kim, Y., Sohn, D., \& Choi, S. M. (2011). Cultural difference in motivations for using social network sites: A comparative study of American and Korean college students. Computers in Human Behavior, 27, 365-372. doi:10.1016/j.chb.2010.08.015 
Kline, R. (2011). Principles and practice of structural equation modeling (3rd ed., Methodology in the social sciences). New York, NY: The Guilford Press.

Kracke, B. (2002). The role of personality, parents and peers in adolescents career exploration. Journal of Adolescence, 25, 19-30. doi:10.1006/jado.2001.0446

LaFontana, K. M., \& Cillessen, A. H. (2002). Children's perceptions of popular and unpopular peers: a multimethod assessment. Developmental Psychology, 38, 635. doi:10.1037//0012-1649.38.5.635

Lee, E., Lee, J.-A., Moon, J. H., \& Sung, Y. (2015). Pictures speak louder than words: Motivations for using Instagram. Cyberpsychology, Behavior, and Social Networking, 18, 552-556. doi:10.1089/cyber.2015.0157

Lockwood, P., \& Kunda, Z. (1997). Superstars and me: Predicting the impact of role models on the self. Journal of Personality and Social Psychology, 73, 91-103. doi: 10.1037/0022-3514.73.1.91

Lup, K., Trub, L., \& Rosenthal, L. (2015). Instagram\# instasad?: Exploring associations among instagram use, depressive symptoms, negative social comparison, and strangers followed. Cyberpsychology, Behavior, and Social Networking, 18, 247-252. doi: $10.1089 /$ cyber.2014.0560

Luthar, S. S., \& Becker, B. E. (2002). Privileged but pressured? A study of affluent youth. Child Development, 73, 1593-1610. doi:10.1111/1467-8624.00492

Madden, M., Lenhart, A., Cortesi, S., Gasser, U., Duggan, M., Smith, A., \& Beaton, M. (2013). Teens, social media, and privacy. (Vol. 21, pp. 2-86). Washington, DC: Pew Research Center. Retrieved from http://www.pewinternet.org/files/2013/05/PIP_TeensSocialMediaandPrivacy_ PDF.pdf 
McCabe, M. P. (2005). The role of performance anxiety in the development and maintenance of sexual dysfunction in men and women. International Journal of Stress Management, 12, 379-388. doi:10.1037/1072-5245.12.4.379

Mendelson, A. L., \& Papacharissi, Z. (2010). Look at us: Collective narcissism in college student Facebook photo galleries In Zizi Papacharissi (Ed.), A Networked Self: Identity, Community and Culture on Social Network Sites (251-273). New York, NY: Routledge.

Muthén, L. K., \& Muthén, B. O. (2017). Mplus User’s Guide (Eight Edition). Los Angeles, CA: Muthén \& Muthén.

Papp, L. M., Danielewicz, J., \& Cayemberg, C. (2012). “Are we Facebook official?” Implications of dating partners' Facebook use and profiles for intimate relationship satisfaction. Cyberpsychology, Behavior, and Social Networking, 15, 85-90. doi:10.1089/cyber.2011.0291

Raacke, J., \& Bonds-Raacke, J. (2008). MySpace and Facebook: Applying the uses and gratifications theory to exploring friend-networking sites. CyberPsychology \& Behavior, 11, 169-174. doi:10.1089/cpb.2007.0056

Ramsey, L. R., \& Horan, A. L. (2017) (in press). Picture this: Women's self-sexualization in photos on social media. Personality and Individual Differences. doi:10.1016/j.paid.2017.06.022

Rumpf, H.-J., Meyer, C., Hapke, U., \& John, U. (2001). Screening for mental health: Validity of the MHI-5 using DSM-IV Axis I psychiatric disorders as gold standard. Psychiatry Research, 105, 243-253. doi:10.1016/S0165-1781(01)00329-8

Russell, S. T. (2005). Conceptualizing positive adolescent sexuality development. Sexuality Research and Social Policy, 2, 4-12. doi:10.1525/srsp.2005.2.3.4Sheldon, P., \& Bryant, K. (2016). Instagram: Motives for its use and relationship to narcissism and 
contextual age. Computers in Human Behavior, 58, 89-97. doi:10.1016/j.chb.2015.12.059

Signorielli, N. (1997). Reflections of girls in the media: A content analysis. A study of television shows and commercials, movies, music videos, and teen magazine articles and ads. Retrieved from ERIC Website:https://files.eric.ed.gov/fulltext/ED444214.pdf

Sprecher, S., \& Metts, S. (1989). Development of the `Romantic Beliefs Scale’ and examination of the effects of gender and gender-role orientation. Journal of Social and Personal Relationships, 6, 387-411. doi:10.1177/0265407589064001

Steinberg, L., \& Morris, A. S. (2001). Adolescent development. Annual Review of Psychology, 52, 83-110. doi:10.1146/annurev.psych.52.1.83

Stice, E., \& Bearman, S.K. (2001). Body-image and eating disturbances prospectively predict increases in depressive symptoms in adolescent girls: A growth curve analyses. Developmental Psychology, 37, 597-607. doi:10.1037//0012-1649.37.5.597

Subrahmanyam, K., \& Šmahel, D. (2011). Digital youth: The role of media in development. New York, NY: Springer.

Thompson, J. K., \& Stice, E. (2001). Thin-ideal internalization: Mounting evidence for a new risk factor for body-image disturbance and eating pathology. Current Directions in Psychological Science, 10, 181-183. doi:10.1111/1467-8721.00144

Tiggemann, M., \& Slater, A. (2014). NetTweens: The internet and body image concerns in preteenage girls. The Journal of Early Adolescence, 34, 606-620. doi:10.1177/0272431613501083

Utz, S., Tanis, M., \& Vermeulen, I. (2012). It is all about being popular: The effects of need for popularity on social network site use. Cyberpsychology, Behavior, and Social Networking, 15, 37-42. doi:10.1089/cyber.2010.0651 
Valenzuela, S., Park, N., \& Kee, K.F. (2009). Is there social capital in a social network site?: Facebook use and college students' life satisfaction, trust, and participation. Journal of Computer-Media Communication, 14, 875-901. doi: 10.1111/j.10836101.2009.01474.x

Vandenbosch, L., \& Eggermont, S. (2012). Understanding sexual objectification: A comprehensive approach toward media exposure and girls' internalization of beauty ideals, self-objectification, and body surveillance: Media, adolescent girls, and selfobjectification. Journal of Communication, 62, 869-887. doi:10.1111/j.14602466.2012.01667.x

Vandenbosch, L., van Oosten, J. M. F., \& Peter, J. (2017). Sexually explicit internet material and adolescents' sexual performance orientation: The mediating roles of enjoyment and perceived utility. Media Psychology, 21, 1-25.

doi:10.1080/15213269.2017.1361842

Vanhaelewyn, B., \& De Marez, L. (2017). Imec.digimeter 2017 measuring digital media trends in Flanders. Retrieved from Imec Website: https://www.imecint.com/nl/digimeter

West, S. G., Taylor, A. B., \& Wu, W. (2012). Model fit and model selection in structural equation modeling In R.H. Hoyle (Ed.), Handbook of structural equation modeling (pp. 209-231). New York, NY: The Guilford Press.

Zywica, J., \& Danowski, J. (2008). The faces of Facebookers: Investigating social enhancement and social compensation hypotheses; predicting Facebook and offline popularity from sociability and self-esteem, and mapping the meanings of popularity with semantic networks. Journal of Computer-Mediated Communication, 14, 1-34. doi:10.1111/j.1083-6101.2008.01429.x 
Table 1

Descriptive statistics per country

\begin{tabular}{|c|c|c|c|c|c|c|}
\hline & Country & $M(S D)$ & $d f$ & $F$ & $p$ & $\eta p^{2}$ \\
\hline \multirow[t]{4}{*}{ Facebook use } & Austria & $1.82(1.39)^{\mathrm{a}}$ & 3 & 80.11 & $p<.001$ & .11 \\
\hline & Belgium & $2.76(1.70)^{\mathrm{b}}$ & & & & \\
\hline & Spain & $1.65(1.44)^{\mathrm{a}}$ & & & & \\
\hline & South Korea & $3.01(1.79)^{\mathrm{b}}$ & & & & \\
\hline \multirow[t]{4}{*}{ Instagram use } & Austria & $3.05(1.58)^{\mathrm{a}}$ & 3 & 204.38 & $p<.001$ & .25 \\
\hline & Belgium & $2.59(1.67)^{\mathrm{b}}$ & & & & \\
\hline & Spain & $4.42(1.86)^{\mathrm{c}}$ & & & & \\
\hline & South Korea & $1.81(1.37)^{\mathrm{d}}$ & & & & \\
\hline \multirow[t]{4}{*}{ The internalization of professional ideals } & Austria & $8.48(1.53)^{\mathrm{a}}$ & 3 & 4.20 & $p<.01$ & .01 \\
\hline & Belgium & $8.44(1.37)^{\mathrm{a}}$ & & & & \\
\hline & Spain & $8.21(1.60)^{\mathrm{a}}$ & & & & \\
\hline & South Korea & $8.18(1.80)^{\mathrm{a}}$ & & & & \\
\hline \multirow[t]{4}{*}{ The internalization of social ideals } & Austria & $6.22(2.28)^{\mathrm{a}}$ & 3 & 62.24 & $p<.001$ & .09 \\
\hline & Belgium & $7.39(1.80)^{\mathrm{b}}$ & & & & \\
\hline & Spain & $7.56(1.89)^{\mathrm{b}}$ & & & & \\
\hline & South Korea & $8.13(1.66)^{\mathrm{c}}$ & & & & \\
\hline \multirow[t]{4}{*}{ The internalization of sexual ideals } & Austria & $6.46(2.90)^{\mathrm{a}}$ & 3 & 70.73 & $p<.001$ & .10 \\
\hline & Belgium & $5.72(2.58)^{\mathrm{b}}$ & & & & \\
\hline & Spain & $6.21(2.45)^{\mathrm{a}}$ & & & & \\
\hline & South Korea & $3.94(2.66)^{\mathrm{c}}$ & & & & \\
\hline \multirow[t]{4}{*}{ The internalization of romantic ideals } & Austria & $7.90(2.35)^{\mathrm{a}}$ & 3 & 22.95 & $p<.001$ & .04 \\
\hline & Belgium & $7.32(2.41)^{\mathrm{b}}$ & & & & \\
\hline & Spain & $6.87(2.53)^{\mathrm{c}}$ & & & & \\
\hline & South Korea & $6.45(2.69)^{\mathrm{d}}$ & & & & \\
\hline \multirow[t]{4}{*}{ Poor mental well-being } & Austria & $2.68(.98)^{\mathrm{a}}$ & 3 & .42 & $p=.74$ & .00 \\
\hline & Belgium & $2.73(.83)^{\mathrm{a}}$ & & & & \\
\hline & Spain & $2.68(.82)^{\mathrm{a}}$ & & & & \\
\hline & South Korea & $2.70(1.05)^{\mathrm{a}}$ & & & & \\
\hline
\end{tabular}


Table 2 (continued)

\begin{tabular}{llccccc}
\hline & Country & $M(S D)$ & $d f$ & $F$ & $p$ & $\eta p^{2}$ \\
\hline Age & Austria & $15.14(1.19)^{\mathrm{a}}$ & 3 & 341.92 & $p<.001$ & \\
& Belgium & $13.72(.67)^{\mathrm{b}}$ & & & \\
& Spain & $15.07(.86)^{\mathrm{a}}$ & & & \\
& South Korea & $14.00(.83)^{\mathrm{c}}$ & & & \\
\hline
\end{tabular}

Note. $M$ and $S D$ with common subscripts do not differ from each other while $M$ and $S D$ with different subscripts differ from each other. For example, $M$ and $S D$ with subscript $a$ differ from means and standard deviations with subscript $b, c$, or $d$. 
Table 2

Descriptive statistics and zero-order correlations (general sample)

\begin{tabular}{|c|c|c|c|c|c|c|c|c|c|c|c|}
\hline & \multicolumn{4}{|c|}{ Descriptive statistics } & \multicolumn{7}{|c|}{ Zero-order correlations } \\
\hline & Min. & Max. & $M$ & $S D$ & 1 & 2 & 3 & 4 & 5 & 6 & 7 \\
\hline 1. Facebook use & 1 & 7 & 2.32 & 1.69 & $\begin{array}{l}-- \\
-1\end{array}$ & & & & & & \\
\hline 2. Instagram use & 1 & 7 & 3.06 & 1.91 & $.13 * * *$ & --- & & & & & \\
\hline 3. The internalization of professional ideals & 1 & 10 & 8.33 & 1.56 & .01 & .04 & --- & & & & \\
\hline 4. The internalization of social ideals & 1 & 10 & 7.37 & 1.99 & $.15 * * *$ & $.11 * * *$ & $.32 * * *$ & --- & & & \\
\hline 5. The internalization of sexual ideals & 1 & 10 & 5.64 & 2.76 & $.05^{*}$ & $.23 * * *$ & $.15^{* * *}$ & $.26 * * *$ & --- & & \\
\hline 6. The internalization of romantic ideals & 1 & 10 & 7.13 & 2.53 & $.08 * *$ & $.08 * * *$ & $.24 * * *$ & $.23 * * *$ & $.58 * * *$ & --- & \\
\hline 7. Poor mental well-being & 1 & 6 & 2.70 & .91 & $.07 * *$ & $.05^{*}$ & -.03 & -.03 & $.08 * *$ & $.08 * * *$ & --- \\
\hline
\end{tabular}

Note. $* p<.05, * * p<.01, * * * p<.001$. 
Table 3

Invariance tests

\begin{tabular}{|c|c|c|c|c|c|c|c|c|}
\hline & \multirow[b]{2}{*}{$\chi^{2}(d f)$} & \multirow[b]{2}{*}{ RMSEA, 90\% CI } & \multirow[b]{2}{*}{ CFI } & \multirow[b]{2}{*}{ TLI } & \multirow[b]{2}{*}{ SRMR } & \multicolumn{3}{|c|}{$\chi^{2}$ difference test } \\
\hline & & & & & & $\chi^{2}$ & $d f$ & $p$ \\
\hline \multicolumn{9}{|c|}{ Measurement invariance tests of internalization scales across countries } \\
\hline Model 1: Configural invariance & $130.57(56)^{* * *}$ & $.05[.04 / .06]$ & .99 & .98 & .03 & & & \\
\hline Model 2: Metric invariance & $174.31(68)^{* * *}$ & $.06[.05 / .07]$ & .98 & .97 & .04 & & & \\
\hline Model 1 vs. Model 2 & & & & & & 43.74 & 12 & $p<.001$ \\
\hline Model 3: Partial invariance & $159.97(67)^{* * *}$ & $.05[.04 / 06]$ & .99 & .98 & .04 & & & \\
\hline Model 1 vs. Model 3 & & & & & & 29.40 & 11 & $p<.01$ \\
\hline Model 4: Partial invariance & $144.49(65)^{* * *}$ & $.05[.04 / .06]$ & .99 & .98 & .03 & & & \\
\hline Model 1 vs. Model 4 & & & & & & 13.92 & 9 & $p=.13$ \\
\hline \multicolumn{9}{|c|}{ Structural invariance test of main model across countries } \\
\hline Unconstrained model & $1688.00(396)^{* * *}$ & $.08[.08 / .09]$ & .88 & .84 & .06 & & & \\
\hline Constrained model & $1749.81(438) * * *$ & $.08[.07 / .08]$ & .87 & .85 & .07 & & & \\
\hline Unconstrained vs. Constrained & & & & & & 61.81 & 42 & $p<.05$ \\
\hline
\end{tabular}

Note. Estimation method = maximum likelihood.

$\chi^{2}=$ Chi-Square; $d f=$ degrees of freedom; RMSEA = root mean square error of approximation; CFI = comparative fit index; TLI = tucker-lewis index; SRMR = standardized root mean square residual.

Measurement invariance: Model $1=$ configural model, multigroup model with no equality constraints; model $2=$ metric model, equality constraints for all factor loadings; model 3 = partial metric model, equality constraints for all factor loadings except for item 2 of South Korea; model 4 = partial metric model, equality constraints for all factor loadings except for item 2 of South Korea and item 11 of South Korea and Austria.

$* * * p<.001$. 
Table 4

Structural invariance test of main model across countries: overview of variant paths

\begin{tabular}{|c|c|c|c|c|c|c|}
\hline Variant path & Country & $\beta$ & $B$ & $S E$ & $p$ & $95 \% C I$ \\
\hline \multirow[t]{4}{*}{ Instagram use and the internalization of social ideals } & Austria & $.26^{\mathrm{a}}$ & .29 & .09 & $<.01$ & $.12 / .43$ \\
\hline & Belgium & $.23^{\mathrm{a}}$ & .18 & .05 & $<.001$ & $.11 / .33$ \\
\hline & Spain & $.27^{\mathrm{a}}$ & .22 & .04 & $<.001$ & $.15 / .37$ \\
\hline & South Korea & $-.06^{\mathrm{b}}$ & -.06 & .08 & .45 & $-.21 / .08$ \\
\hline \multirow[t]{4}{*}{ Instagram use and poor mental well-being } & Austria & $.09^{\mathrm{a}}$ & .03 & .03 & .38 & $-.08 / .26$ \\
\hline & Belgium & $.10^{\mathrm{a}}$ & .03 & .02 & .07 & $-.01 / .20$ \\
\hline & Spain & $-.04^{\mathrm{ab}}$ & -.01 & .01 & .41 & $-.14 / .06$ \\
\hline & South Korea & $-.15^{b}$ & -.12 & .06 & $<.05$ & $-.31 /-.01$ \\
\hline \multirow[t]{4}{*}{ Facebook use and the internalization of professional ideals } & Austria & $.14^{\mathrm{a}}$ & .09 & .05 & $<.05$ & $03 / .30$ \\
\hline & Belgium & $-.07^{\mathrm{b}}$ & -.03 & .03 & .25 & $-.21 / .04$ \\
\hline & Spain & $-.02^{\mathrm{ab}}$ & -.02 & .05 & .74 & $-.10 / .20$ \\
\hline & South Korea & $-.02^{\mathrm{ab}}$ & -.02 & .07 & .80 & $-.17 / .12$ \\
\hline \multirow[t]{4}{*}{ Facebook use and the internalization of social ideals } & Austria & .19 & .24 & .10 & $<.05$ & $.05 / .34$ \\
\hline & Belgium & .05 & .04 & .05 & .44 & $-.08 / .17$ \\
\hline & Spain & -.06 & -.06 & .05 & .23 & $-.16 / .03$ \\
\hline & South Korea & .26 & .19 & .05 & $<.001$ & $.13 / .38$ \\
\hline \multirow[t]{4}{*}{ Facebook use and the internalization of sexual ideals } & Austria & $.06^{\mathrm{ab}}$ & .12 & .13 & .34 & $-.08 / .17$ \\
\hline & Belgium & $.16^{\mathrm{a}}$ & .22 & .07 & $<.001$ & $.08 / .25$ \\
\hline & Spain & $.01^{\mathrm{b}}$ & .02 & .07 & .75 & $-.07 / .10$ \\
\hline & South Korea & $.14^{\mathrm{ab}}$ & .19 & .05 & $<.05$ & $-.00 / .29$ \\
\hline \multirow[t]{4}{*}{ Facebook use and poor mental well-being } & Austria & $-.01^{\mathrm{ab}}$ & -.01 & .03 & .88 & $-.16 / .15$ \\
\hline & Belgium & $.02^{\mathrm{a}}$ & .01 & .02 & .78 & $-.09 / .13$ \\
\hline & Spain & $.13^{\mathrm{b}}$ & .05 & .02 & $<.05$ & $.02 / .24$ \\
\hline & South Korea & $.14^{\mathrm{ab}}$ & .08 & .05 & .09 & $-.03 / .30$ \\
\hline
\end{tabular}

Note. Paths with common subscripts do not differ from each other (e.g., paths with subscript $a b$ do not differ from paths with subscript $a, b$, and $a b$ ) while paths with different subscripts differ from each other (e.g., paths with subscript $a$ differ from paths with subscript $b$ ). Differences in the relations between Facebook use and the internalization of social ideals were too complex to mark with subscripts: Austria differs significantly from Spain. Belgium differs significantly from South Korea. Spain differs significantly from South Korea. Austria does not differ significantly from Belgium and South Korea. Belgium does not differ significantly from Austria and Spain. Spain does not differ significantly from Belgium. 
H4

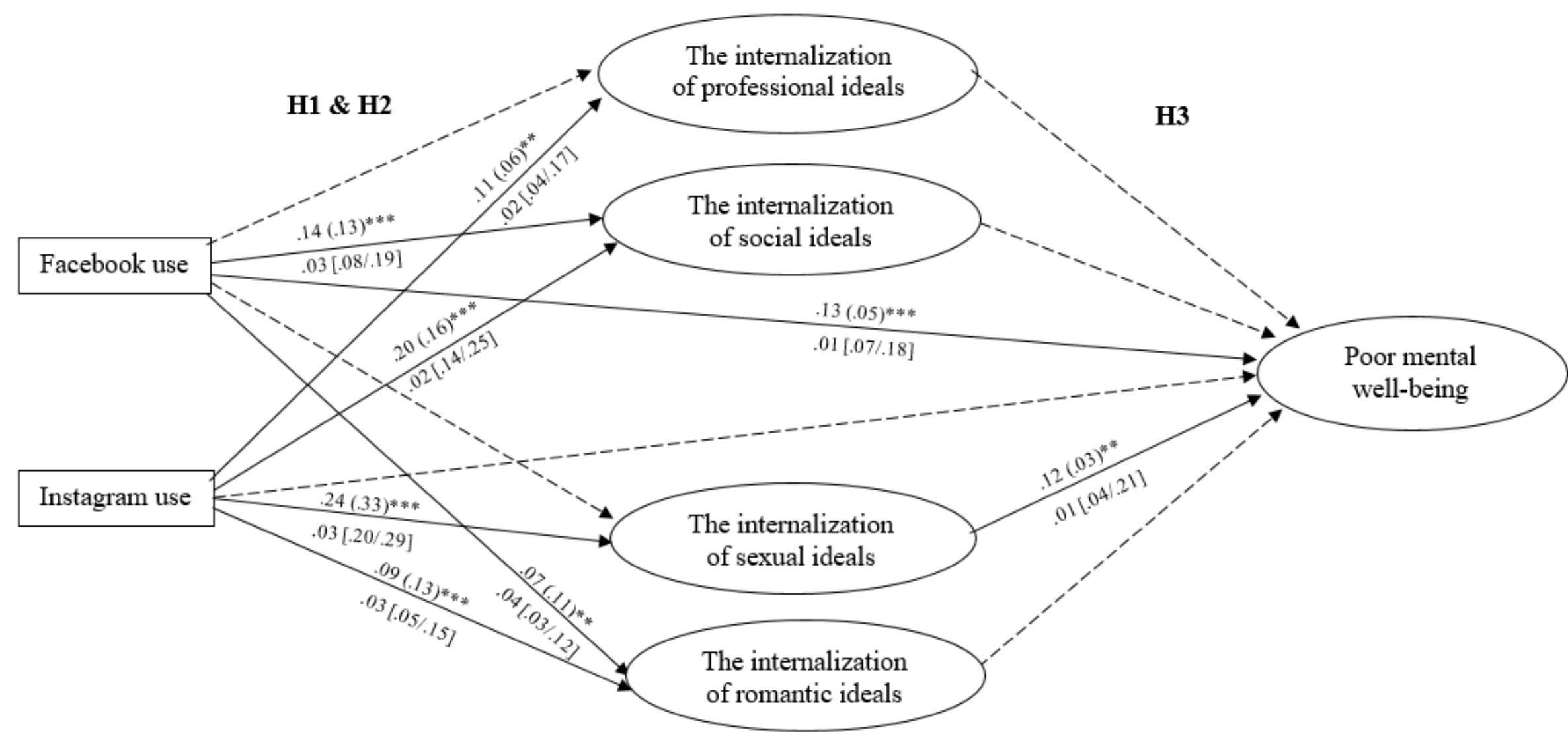

Figure 1. Structural equation model of the relations between Facebook use, Instagram use, the internalization of professional, social, sexual, and romantic ideals, and poor mental well-being across countries $(\mathrm{N}=1,983)$.

$\chi^{2}(87)=724.60, p<.001, \mathrm{RMSEA}=.06(90 \% \mathrm{CI}: .06 / .07), \mathrm{CFI}=.94, \mathrm{TLI}=.90, \mathrm{SRMR}=.04$

Note. Above the arrow: First value reflect standardized coefficient (beta), value within brackets reflects unstandardized coefficients (b-value).

Below the arrow: First value reflects standard errors, values within squared brackets reflect $95 \%$ confidence intervals.

Only significant coefficients are shown $\left({ }^{*} p<.05, * * p<.01, * * * p<.001\right)$; dashed lines are non-significant paths. 


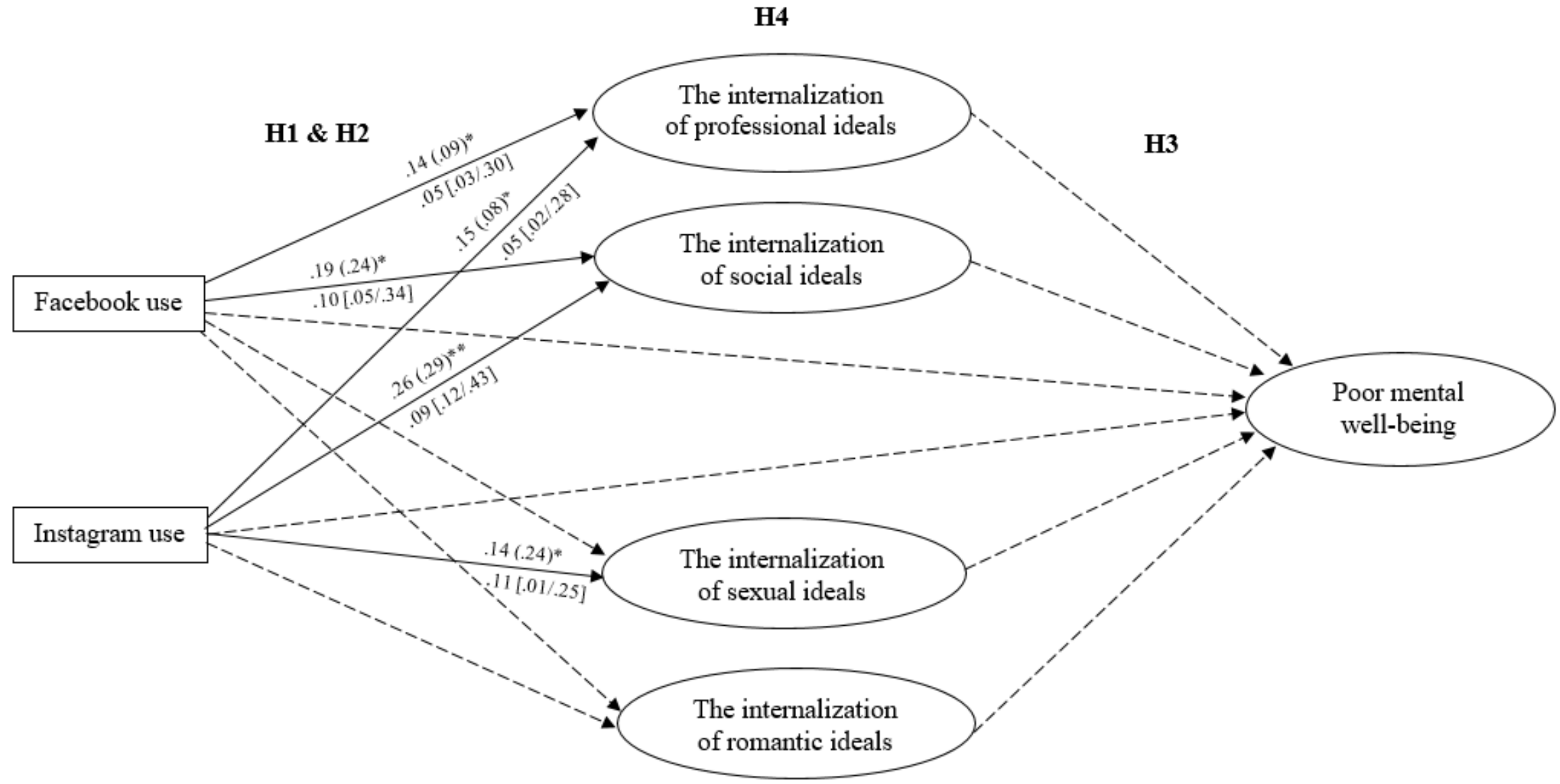

Figure 2. Structural equation model of the relations between Facebook use, Instagram use, the internalization of professional, social, sexual, and romantic ideals, and poor mental well-being in Austria $(\mathrm{N}=368)$.

$\chi^{2}(87)=188.36, p<.001, \mathrm{RMSEA}=.06(90 \% \mathrm{CI}: .05 / .07), \mathrm{CFI}=.94, \mathrm{TLI}=.91, \mathrm{SRMR}=.04$

Note. Above the arrow: First value reflect standardized coefficient (beta), value within brackets reflects unstandardized coefficients (b-value).

Below the arrow: First value reflects standard errors, values within squared brackets reflect $95 \%$ confidence intervals.

Only significant coefficients are shown $\left({ }^{*} p<.05\right.$, ** $p<.01$, *** $\left.p<.001\right)$; dashed lines are non-significant paths. 


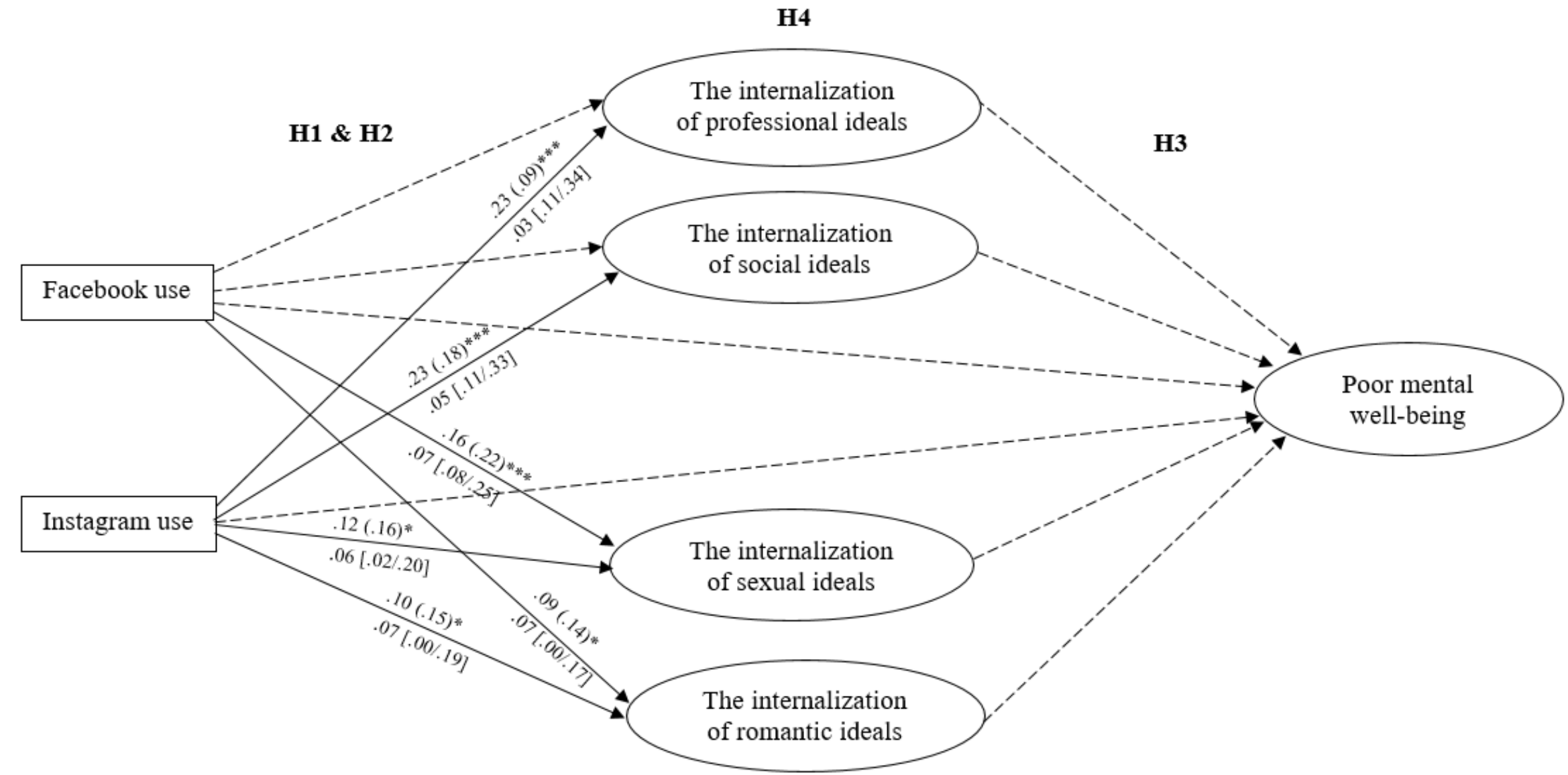

Figure 3. Structural equation model of the relations between Facebook use, Instagram use, the internalization of professional, social, sexual, and romantic ideals, and poor mental well-being in Belgium $(\mathrm{N}=675)$.

$\chi^{2}(87)=252.79, p<.001, \mathrm{RMSEA}=.05(90 \% \mathrm{CI}: .05 / .06), \mathrm{CFI}=.95, \mathrm{TLI}=.93, \mathrm{SRMR}=.04$.

Note. Above the arrow: First value reflect standardized coefficient (beta), value within brackets reflects unstandardized coefficients (b-value).

Below the arrow: First value reflects standard errors, values within squared brackets reflect $95 \%$ confidence intervals.

Only significant coefficients are shown $(* p<.05, * * p<.01, * * * p<.001)$; dashed lines are non-significant paths. 


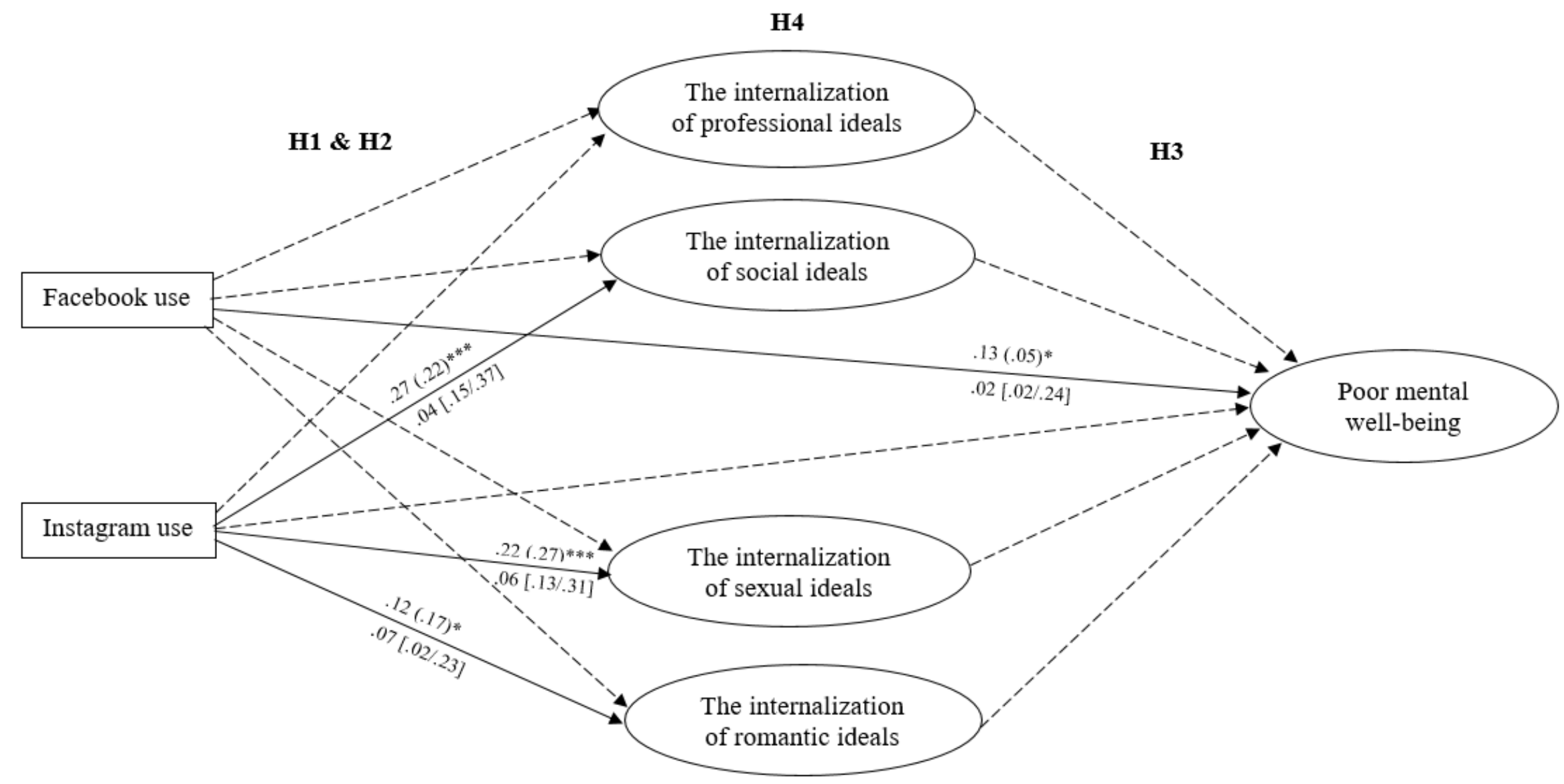

Figure 4. Structural equation model of the relations between Facebook use, Instagram use, the internalization of professional, social, sexual, and romantic ideals, and poor mental well-being in Spain $(\mathrm{N}=564)$.

$\chi^{2}(87)=274.86, \mathrm{p}<.001, \mathrm{RMSEA}=.06(90 \% \mathrm{CI}: .05 / .07), \mathrm{CFI}=.93, \mathrm{TLI}=.90, \mathrm{SRMR}=.04$.

Note. Above the arrow: First value reflect standardized coefficient (beta), value within brackets reflects unstandardized coefficients (b-value).

Below the arrow: First value reflects standard errors, values within squared brackets reflect $95 \%$ confidence intervals.

Only significant coefficients are shown $(* \mathrm{p}<.05, * * \mathrm{p}<.01, * * * \mathrm{p}<.001)$; dashed lines are non-significant paths. 


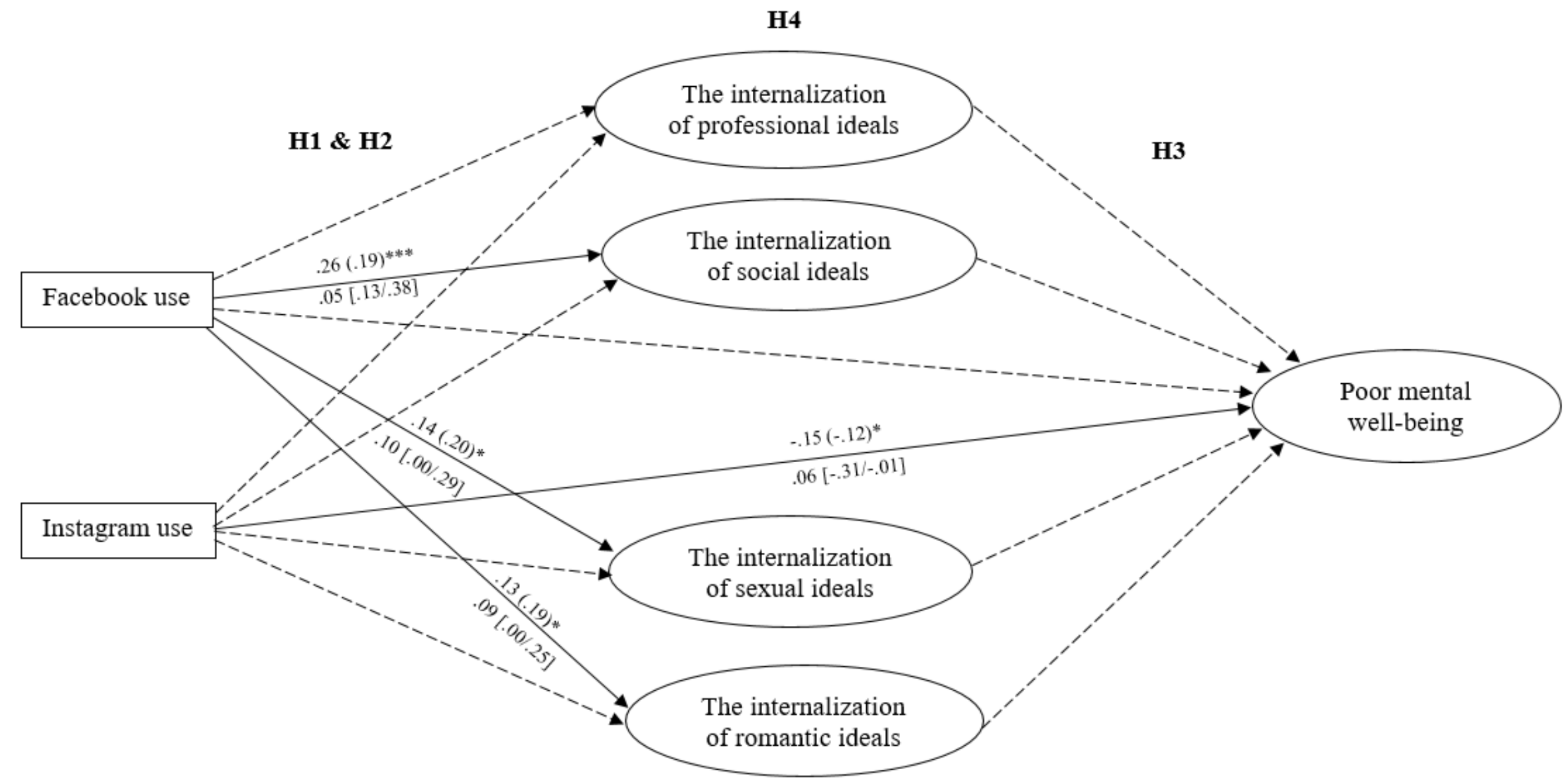

Figure 5. Structural equation model of the relations between Facebook use, Instagram use, the internalization of professional, social, sexual, and romantic ideals, and poor mental well-being in South Korea $(\mathrm{N}=376)$.

$\chi^{2}(87)=262.04, p<.001$, RMSEA $=.07(90 \%$ CI: $.06 / .08)$, CFI $=.93$, TLI $=.90$, SRMR $=.05$.

Note. Above the arrow: First value reflect standardized coefficient (beta), value within brackets reflects unstandardized coefficients (b-value).

Below the arrow: First value reflects standard errors, values within squared brackets reflect $95 \%$ confidence intervals.

Only significant coefficients are shown $(* \mathrm{p}<.05, * * \mathrm{p}<.01, * * * \mathrm{p}<.001)$; dashed lines are non-significant paths. 